\title{
Model of creation a multilayer structure for production of building pipes
}

\author{
Valery Dyadichev ${ }^{1}$, Andrey Kolesnikov ${ }^{2}$, Aleksandr Dyadichev ${ }^{1}$, Sergey Menyuk ${ }^{1}$ and \\ Ekaterina Dyadicheva ${ }^{1}$ \\ ${ }^{1}$ V.I. Vernadsky Crimean Federal University, Academy of Construction and Architecture, \\ Engineering Center «Bio-positive Construction and Resource-Saving», Pavlenko Str. 3, Simferopol, \\ Crimea, 295000, Russia \\ ${ }^{2}$ Lugansk National University named after Vladimir Dal, Department of Automation and Computer- \\ Integrated Technologies, Molodegniy sq. 20-A, Lugansk, 91034, Ukraine
}

\begin{abstract}
A scientific article presents the main goals that can be achieved through the use of co-extrusion processing technology and equipment for the processing of secondary polymer and building materials. The main indicator of the multilayered product being created in the process of coextrusion processing of secondary building and polymer materials is determined. The methods of quality control of the mixture preparation from secondary materials and quality of manufacturing of a finished coextrusion product are considered. A reference mathematical model of a coextruded product containing secondary building and polymer materials is constructed. Based on the results of the studies, the article presents an analysis of the ecological state of building and polymer wastes. The necessity of further research in the field of improving the model of the formation of a multilayer structure of a given quality for the process of processing this waste is substantiated. For the existing practice of preparing a mixture of polymer and building material wastes, the volumetric costs of components are determined by recipes. An analysis of the equations obtained allows us to refine the recommended numerical values of the percentage of primary polymer on the basis of the required standard strength of the building pipe from secondary materials.
\end{abstract}

\section{Introduction}

The large-scale development of the construction industry requires the creation of new building materials that will be in demand for the creation of buildings and structures. The determining influence in this case is the cost of raw materials, as well as environmental and resource factors. Thus, by reducing the cost of raw materials, it is possible to reduce the price of housing construction, and, consequently, to solve the problem of housing availability. The process of construction is inevitably associated with the emergence of waste (broken brick, cinder block, remains of concrete and other building structures). These wastes are very laborious and energy intensive for processing and, as a rule, is not processed at all. Thus, there is an environmental problem associated with the disposal of building waste. At the same time, these materials can serve as valuable raw materials in 
case of finding a successful method of processing them, which allows to return the data to the material in the resource base of construction sites. This will allow, in this way, to solve the resource problem of new building materials having a significantly lower cost due to the use of secondary building materials in their structure.

Co-extrusion processing of secondary building and polymer materials is a promising technology that allows producing new useful products of wastes [1-5].

The area of secondary processing of polymeric materials is also an actual problem. The authors obtained significant results in the development of technology and equipment for coextrusion processing of secondary polymer [6-8]. However, a significant drawback in the results of the work carried out was the use of the obtained secondary multilayered polymerbased constructions $[9,10]$.

Co-processing of polymer and building materials is a promising area of application of co-extrusion technology, since the requirements in this field for materials have mainly strength characteristics $[11,12]$.

Thus, the authors propose the use of co-extrusion technology for the production of a multilayer polymer product of the building sphere (pipe, reinforcing bar, boxes, beams and other profile building materials), which includes a layer of secondary building materials, secondary polymer materials, primary polymer, fillers, dyes, stabilizers and plasticizers $[13,14]$.

A significant problem in obtaining such a multilayer structure is the development of a quality assessment criterion and the formation of a structure preparation model for coextrusion processing of secondary building and polymer materials [15, 16]. Optimal construction of the structure makes it possible to save its most expensive components - the primary polymer and the filler [1], the excess content of which makes the technology ineffective due to the high cost of a new product $[17,18]$.

The purpose of the study is to formulate a criterion for assessing the quality and model of preparation of a multilayer structure for manufacturing building pipes with the use of secondary materials by the co-extrusion method.

Objectives of the study:

1. Determine the main indicator of the multilayer product being created in the process of co-extrusion processing of secondary building and polymer materials;

2. Conduct an analysis of quality control methods for the preparation of mixtures from secondary materials and the quality of the finished co-extrusion product;

3. Construct a reference mathematical model of a co-extruded product containing secondary building and polymer materials;

4. Create a model for the preparation of a multilayer structure of a given quality for the process of processing secondary building and polymer materials.

\section{Methods}

When designing the structures of co-extrusion machines for processing secondary building and polymeric materials with the manufacture of a multilayer structure, the normative strength of the middle layer of the co-extruded product is used as the main indicator of the multilayered product being created, the normative strength of the middle layer of the coextruded product is used. It is formed from building and polymeric material wastes and has the largest thickness of all layers. The normative strength is the value of the time resistance for uniaxial stretching of samples of the middle layer mixture. The effectiveness of using multi-layer structures for waste disposal of these materials largely depends on the accuracy and efficiency of determining this indicator.

To assess quickly the quality of the mixture during its preparation, a method that provides the creation of reference mathematical models of the co-extruded product and the 
determination of its parameters was developed. The reference model of the multilayer structure, unlike the structure recipe, displays the empirical relationships connecting the composition and properties of the multilayer structure, and it is represented as a function of the goal and constraints.

The reference mathematical model of the co-extruded product is used to compare its parameters with the parameters of the mixture for the multilayer structure obtained during the preparation. Based on the results of comparing the values of the composition of the components and the properties of the mixture, it is possible to predict the strength characteristics of a multilayer product, to determine the reasons for the deviation of the parameters, and to influence the technology of preparation of the mixture. Such approach allows not only to obtain an operative assessment of the quality of the mixture, but also to choose a method for controlling the process of its production.

Thus, according to the accepted criterion, the basic equation of the reference mixture model for the multilayer structure (target function) should reflect the dependence of the required primary polymer $m_{p p}$ flow rate on the composition of the remaining components (building filled waste $m_{t w}$, a mixture of secondary polymer $m_{s p \text { : filler }} m_{f}$ ) in order to ensure homogeneity mixture for the established standard strength of the mixture for the coextruded product $R_{N}(1)$ :

$$
m_{p p}^{3}=f\left(m_{f w}, m_{s p}, m_{f}, R_{N}\right) .
$$

Since the obligatory condition for the preparation of the co-extruded product is to ensure the normative strength of the created multilayer product and the corresponding rheological characteristics of the mixture for the multilayer structure, it is logical to use the minimum permissible strength $R_{\text {perm }}$ of the mixture for co-extrusion and one of the rheological characteristics of the mixture for the multilayer structure as constraints in the mixture model for a multilayer structure. The presence of the latter as constraints in the mixture model for a multilayer structure is mandatory, because, in consistency, the mixture must provide minimal resistance to movement when it is transported through the forming co-extrusion die channels from the extruders to the exit from the die, and, also fill the die channels with maximum fullness.

It is known that the rheological properties of structured mixtures are determined by two parameters: viscosity $\eta_{\text {and critical shear stress }} \tau_{0}$.

The viscosity of the mixture from polymer wastes is optimal if the mixture prepared from polymer waste has the minimum necessary mobility (plasticity). The optimum plasticity of the mixture Pm can be established as a function of the dynamic viscosity $\eta_{\text {and }}$ the magnitude of the tangential stresses arising when the mixture is moved through the channels of the extruder and co-extrusion die channels, i.e. $\operatorname{Pm}=\mathrm{f}\left({ }^{\eta}, \tau\right)$.

The optimum plasticity of the mixture, satisfying the requirements of transportability, is in the range from 1 to $6 \mathrm{~g} / 10 \mathrm{~min}$. Such plasticity is achieved by using a filler-binding ratio from 0.2 to 0.6 , i.e. at the filler consumption from 200 to $600 \mathrm{~m}^{3}$ per $1000 \mathrm{~m}^{3}$ of the mixture. With a filler consumption of more than $600 \mathrm{~m}^{3}$, the mixture turns out to be excessively viscous and non-transportable, and at a filler consumption of less than $200 \mathrm{~m}^{3}$, the physico-mechanical properties affecting the plasticity of the mixture deteriorate.

The critical shear stress $\tau_{0}$ is the force that must be applied to the mixture so that its flow begins. In the future, the flow takes place according to the law of a viscous liquid, as in the absence of a structure. The behavior of structured suspensions in general form could be determined by the Shvedov-Bingham equation, with reference to viscoplastic systems (2): 


$$
\tau-\tau_{0}=\eta_{p l} \cdot \frac{d v}{d r}
$$

where: $\eta_{p l}$ - a coefficient of plastic viscosity; $\frac{d v}{d r}$ - a velocity gradient.

The critical shear stress $\tau_{0}$ also depends strongly on the filler-binding ratio used. Based on the conditions for co-extruding the mixture into a multilayer structure and the fact that the mixture is transported through the extruder and co-extrusion die channels, it is advisable to assume that the critical shear stress $\tau_{0}$ of the prepared mixture should be less than the maximum permissible $\tau_{0 \text { perm }}$, i.e. there is a limitation in terms of reducing the filler consumption, and use the maximum permissible shear stress $\tau_{0 \text { perm }}$ and shear stress $\tau_{0}$ as a mixture parameter.

The choice is stopped on this rheological characteristic of the mixture, as the most important from the viewpoint of preventing the formation of plugs and channel plugs (in co-extrusion equipment, depending on the size and design of the extruder and the die, the value is regulated $\tau_{0 \text { perm }}$, and also because of the simplicity and convenience of measuring this characteristic in laboratory conditions.

The primary polymer consumption $m_{p p}^{3}$, the normative strength of the created product $R$, the critical shear stress $\tau_{0}$ for the mixture, acting as parameters of the mixture, depend on the following factors: a ratio of the volumetric mass of the components in $1 \mathrm{~m}^{3}$ of the mixture (filled waste, $m_{f w}$; secondary raw materials (polymer and building waste), $m_{s r m}$; primary polymer, $m_{p p}$; filler, $m_{f}$ ); a normative strength of a multilayer product, $R$; a set critical shear stress, $\tau_{0}$; a specific surface area of the filler material, $S$; a filler-binding ratio, $\frac{m_{f}}{m_{p p}}$ for mixtures based on the primary polymer, $\frac{m_{f}}{m_{f w}}$ for complex binder; a granulometric composition of active and inert fillers, $q_{f w}$ and $q_{s p}$.

The necessary volume mass $m_{p p}^{3}$ of the primary polymer, the strength of the product $R$ and the critical shear stress $\tau_{0}$ can be represented as a function of several variables $Z_{i}(3)-$ (5):

$$
\begin{aligned}
R & =f_{1}\left(z_{1 R}, \ldots, z_{n R}\right), \\
\tau_{0} & =f_{2}\left(z_{1 \tau_{0}}, \ldots, z_{n \tau_{0}}\right), \\
m_{p p}^{3} & =f_{3}\left(z_{1 p p}, \ldots, z_{n p p}\right) .
\end{aligned}
$$

If as a function of the objective use the determination of the volume mass of the primary polymer $m_{p p}^{3}$ that is necessary for preparing a mixture of polymer and building material wastes with the critical shear stress $\tau_{0}$ and forming a co-extruded product of strength $R$ depending on the actual masses of the remaining components, their granulometric composition and specific surface of the filler, conditions for ensuring the normative strength of co-extrusion $R>R_{\text {perm }}$ and the critical shear stress of the mixture $\tau_{0} \leq \tau_{0 \text { perm }}$ should be used as constraints. 
Therefore, it is possible to construct a model of a mixture of the form:

$$
\begin{aligned}
m_{p p}^{3}= & f\left(m_{s r m}, m_{f w}, m_{f}, q_{s r}, q_{f w}, S, R_{N}\right), \\
R & \geq R_{p e r m}, \\
& \tau_{0} \leq \tau_{0 \text { perm }} .
\end{aligned}
$$

The study of the parameters of the mixture using mathematical modeling methods is expedient to carry out initially for some of the determining factors, confining ourselves only to study the effect of component costs on the parameters of the mixture. This allows us, essentially simplifying the model, to reveal the main regularities existing between the factors and parameters of the mixture. Then, at the stage of verification and practical implementation of the model, appropriate corrections to the model can be introduced, taking into account the influence of other factors. This approach is also justified, in connection with the complexity of the operational control of the granulometric composition of the components and the critical surface of the filler.

With the current practice of preparing a mixture of polymer and building material wastes, the volumetric costs of the components are set according to the recipes recommended for obtaining the mixture (multilayered product) of the required strength (15.5 MPa, 21.5 MPa, 33.0 MPa, 55.0 MPa) in accordance with the layer thickness and secondary polymers and building materials (Figure 1).

Therefore, the development of reference mathematical models of the mixture should be carried out for the same, fixed values of the normative co-extrusion strength. This makes it possible, by narrowing the range of change of factors, to describe the existing relationships using lower-order models. The latter greatly simplifies the organization of the experiment and the processing of results, improves the reliability of the obtained characteristics.

The solution of model (6) - (8) will allow an indirect estimation of each dimension $m_{p p}^{3}$, $R, \tau_{0}$ and determine the influence of each factor $\left(m_{s r m}, m_{f w}, m_{f w}\right)$ on the primary polymer consumption. This makes it possible to correct the consumption of the primary polymer when any factor changes, maintaining the homogeneity of the mixture.

The reference mathematical models of a mixture of polymer and building material wastes are developed for the most commonly used types of mixture: primary polymer + mixture of secondary wastes of polymers and building materials, waste of construction and polymer materials with filler + primary polymer + mixture of secondary wastes of polymers and building materials.

To determine the equations of the mixture model: wastes of polymers and building materials with filler + primary polymer + a mixture of secondary wastes of polymers and building materials, the coefficients of which approximate the properties of the actual dependence $m_{p p}^{3}=f\left(m_{s r m}, m_{f w}, m_{f}, R_{N}\right)$ existing in the object in the vicinity of some combination of arguments (factors) $m_{s r m 0}, m_{f w 0}, m_{f 0}$, that are called the based, the statistical method of experiment planning (method of full factorial experiment) is used.

Then the general form of the regression equations for the developed mixture model is:

$$
\begin{aligned}
m_{p p}^{3}=a_{0 p p} \cdot x_{0} & +a_{1 p p} \cdot x_{1}+a_{2 p p} \cdot x_{2}+\ldots \\
& +a_{n p p} \cdot x_{n} \\
R=a_{0 R} \cdot x_{0} & +a_{1 R} \cdot x_{1}+a_{2 R} \cdot x_{2}+\ldots \\
& +a_{n R} \cdot x_{n}
\end{aligned}
$$




$$
\begin{gathered}
\tau_{0}=a_{0 \tau_{0}} \cdot x_{0}+a_{1 \tau_{0}} \cdot x_{1}+a_{2 \tau_{0}} \cdot x_{2}+\ldots \\
+a_{\Pi \tau_{0}} \cdot x_{\Pi}
\end{gathered}
$$

where: $a_{i j}$ - coefficients of regression equations $\left(i=0,1, \ldots n ; j=p p, R, \tau_{0}\right) ; x_{i}$ - factors that

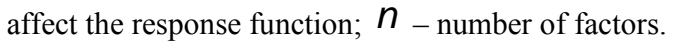

A A

A A

PE SRM PE

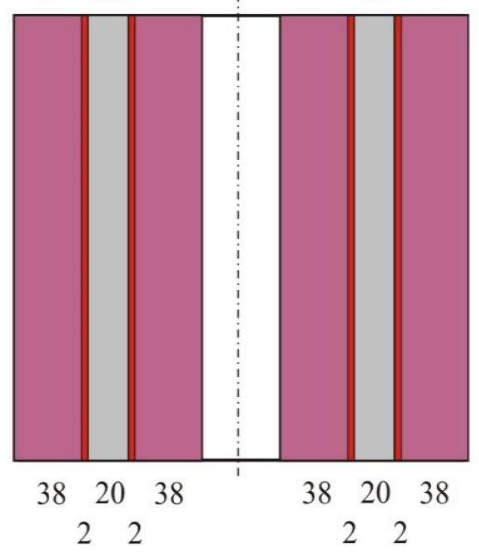

Composition 1 - Pipes for transportation of granular substances of different chemical composition $-55 \mathrm{MPa}$

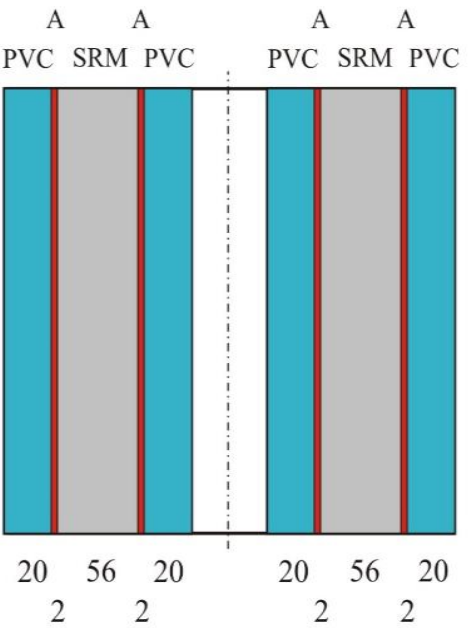

Composition 3 - Pipes for protective communications and engineering facilities $-21.5 \mathrm{MPa}$
A A

A A

PA SRM PA PA SRM PA

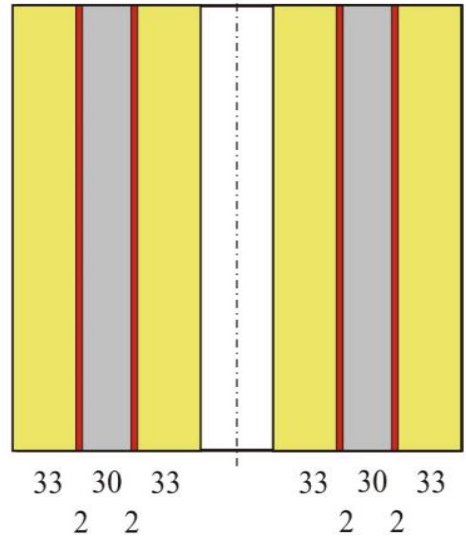

Composition 2 - Pipes for transportation of liquids and gases $-33 \mathrm{MPa}$

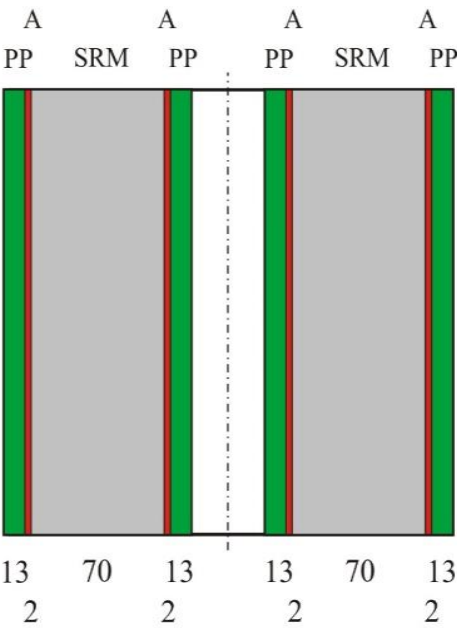

Composition 4 - Pipes for the construction of buildings and structures $-15.5 \mathrm{MPa}$

Fig. 1. Multilayer compositions with co-extrusion with secondary polymeric and building materials: PE - polyethylene, SRM - secondary raw materials, PA - polyamide, PVC - polyvinylchloride, PP polypropylene, A - adhesive.

The mixture model: primary polymer + a mixture of secondary wastes of polymers and building materials differs from the mixture: waste of polymers and building materials with filler + primary polymer + a mixture of secondary wastes of polymers and building 
materials by the values of the coefficients of equations $a_{i j}$ and the absence of a factor corresponding to the component filled wastes of polymers and building materials.

\section{Results and Discussion}

To obtain a reference model of such a mixture, we carried out the full factorial experiment $2^{\mathrm{n}}$, where $\mathrm{n}=4$, for the most widely used formulations, according to the method described above.

Equations (9) - (11) establish the relationships between the mass of the primary polymer $_{p p}^{3}$, as the most expensive component, and the masses of the remaining components $\left(m_{s r m}, m_{f}, m_{f w}\right)$ per $1 \mathrm{~m}^{3}$ of the mixture, at which the necessary normative strength of the coextruded article and the required mobility of the mixture are provided. That is, the data of the group of equations are models for calculating reference values $m_{p p}^{3}$ that can be used to determine the design tasks for the primary polymer consumption with automated quality management of the mixture if the minimum consumption of this component is considered as the control objective.

An analysis of the equations obtained makes it possible to refine the numerical values $m_{p p}^{3}$

recommended by the recipe. Thus, to obtain a coextruded mixture of $33.0 \mathrm{MPa}$, it is sufficient to use $38 \mathrm{~kg}$ of primary polymer per $1 \mathrm{~m}^{3}$ of the mixture instead of $40 \mathrm{~kg}$ recommended by the recipe.

Table 1 lists the values $m_{p p}^{3}$ recommended by the recipe and calculated by the model, under which the required regulatory strength of the product is ensured. With large volumes of co-extrusion (about 10 thousand $\mathrm{m}^{3} /$ year), the economy of the primary polymer can amount to an impressive figure (approximately 30 tons/ year with the mixture: primary polymer + a mixture of secondary waste and 20 tons/ year with mixture: filled waste + primary polymer + a mixture of secondary waste).

Table 1. Recommended values of primary polymer mass per $1 \mathrm{~m}^{3}$ of co-extrusion mixture.

\begin{tabular}{|c|c|c|c|c|c|c|}
\hline \multirow{2}{*}{ R, MPa } & \multicolumn{3}{|c|}{$\begin{array}{c}\text { Primary polymer + a mixture } \\
\text { of secondary waste }\end{array}$} & \multicolumn{2}{|c|}{$\begin{array}{c}\text { Wastes with filler + primary polymer + a } \\
\text { mixture of secondary waste }\end{array}$} \\
\cline { 2 - 7 } & Recipe, $\mathrm{kg}$ & Model, $\mathrm{kg}$ & Excess & Recipe, $\mathrm{kg}$ & Model, $\mathrm{kg}$ & Excess \\
\hline 55.0 & 210 & 208.99 & 1.01 & 80 & 80.61 & -0.61 \\
\hline 33.0 & 130 & 126.92 & 3.08 & 40 & 37.95 & 2.05 \\
\hline 21.5 & 70 & 68.21 & 1.79 & 20 & 18.6 & 1.4 \\
\hline
\end{tabular}

\section{Conclusions}

1. The main indicator of the multilayer product being created in the process of coextrusion processing of secondary building and polymer materials is the normative strength of the middle layer of the co-extrusion product.

2. The methods of controlling the quality of preparation of a mixture from secondary materials and the quality of manufacturing of a finished co-extrusion product are 
considered. The reference mathematical model of a co-extruded product containing secondary building and polymer materials is constructed.

3. The reference mathematical models of a mixture of polymer and building material wastes have been developed for the most commonly used types of mixture: primary polymer + a mixture of secondary wastes of polymers and building materials, waste of polymers and building materials with filler + primary polymer + a mixture of secondary wastes of polymers and building materials.

4. For the current practice of preparing a mixture of polymer and building material wastes, the volumetric costs of the components are determined according to the recipes recommended for obtaining the mixture (multilayered product) of the required strength (15.5 MPa, 21.5 MPa, 33.0 MPa, 55.0 MPa) in accordance with the layer thickness and existing secondary polymers and building materials.

The study was carried out with the financial support of the Ministry of Education and Science of the Russian Federation within the framework of the scientific project 10.1622.2017/4.6.

\section{References}

1. C. Rauwendaal, Adv. Polym. Techn., 11, 19-25 (1992)

2. C. Rauwendaal, J.F. Ingen Housz, J Reinf. Plast. Comp., 9, 583-601 (1990)

3. G.A.Bystrov, V. Galperin, B.P. Titov, Neutralization and utilization of plastics waste in the plastics industry (Leningrad, 1982)

4. V.V Dyadichev, B.N. Lokotosh, V.V. Levanichev, Modeling coextrusion process (Lugansk, 1998)

5. V.V. Dyadichev, Processing of waste polymeric materials by coextrusion (Lugansk, 2003)

6. V.V. Dyadichev, Extrusion of secondary polymers (Lugansk, 2003)

7. D.F. Kagan, V.E. Gul, L.D. Samarin, Multilayer and combined film materials, (Moscow, 1989)

8. G.D. Kalynovskaya, Waste processing routes for laminates (St. Petersburg, 1997)

9. P. Reitemeyer, Kunststoffe, 5, 395-397 (1988)

10. F.R. Nissel, Coextrusion V, SPE Regional Technical Conference (Arlington, 1989)

11. J. Perdikonlias, J. Petric, PM \& E, 9, 35-39 (1992)

12. A. Auffermenn, VDI, Verheg, 11-32 (Dussldorf, 1990)

13. R.J. Nichols, F. Kheradi, Modern Plastics (1984)

14. K. Luker, Paper presented at the Continuous Compounding Conference (Beachwood, Ohio, 2000)

15. M. Shahinpoor, J Nonnewton Fluid Mech., 12, 31-38 (1983)

16. F.J. Brinkschroeder, F. Johannaber, Kunststoffe, 71, 138-143 (1981)

17. B. Davis, Polym. Eng. Sci., 38 (7), 1199 (1998)

18. C.J. Rauwendaal, Screw Extruder with Adjustable Groove Depth., U.S. Patent 5,909,958 (1999) 